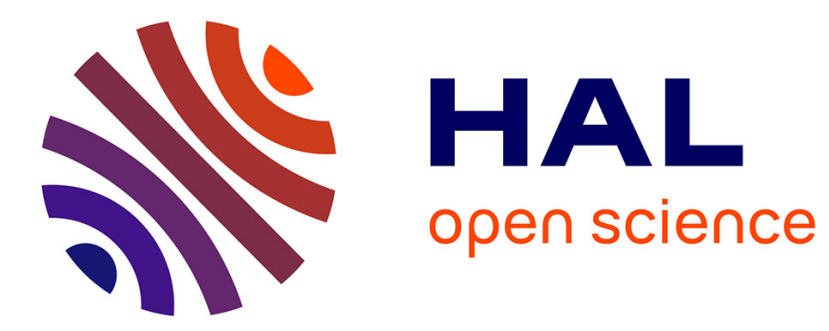

\title{
Convergence to equilibrium in competitive Lotka-Volterra and chemostat systems
}

Nicolas Champagnat, Pierre-Emmanuel Jabin, Gael Raoul

\section{To cite this version:}

Nicolas Champagnat, Pierre-Emmanuel Jabin, Gael Raoul. Convergence to equilibrium in competitive Lotka-Volterra and chemostat systems. Comptes rendus de l'Académie des sciences. Série I, Mathématique, 2010, 348 (23-24), pp.1267-1272. 10.1016/j.crma.2010.11.001 • inria-00495991

\section{HAL Id: inria-00495991 https://hal.inria.fr/inria-00495991}

Submitted on 10 Mar 2016

HAL is a multi-disciplinary open access archive for the deposit and dissemination of scientific research documents, whether they are published or not. The documents may come from teaching and research institutions in France or abroad, or from public or private research centers.
L'archive ouverte pluridisciplinaire HAL, est destinée au dépôt et à la diffusion de documents scientifiques de niveau recherche, publiés ou non, émanant des établissements d'enseignement et de recherche français ou étrangers, des laboratoires publics ou privés. 


\title{
Convergence to equilibrium in competitive Lotka-Volterra and chemostat systems
}

\author{
Nicolas Champagnat ${ }^{1}$, Pierre-Emmanuel Jabin ${ }^{1,2}$, Gaël Raoul ${ }^{3}$
}

\begin{abstract}
We study a generalized system of ODE's modeling a finite number of biological populations in a competitive interaction. We adapt the techniques in [8] and [2] to prove the convergence to a unique stable equilibrium.

Résumé. Nous étudions un système généralisé d'équations différentielles modélisant un nombre fini de populations biologiques en interaction compétitive. En adaptant les techniques de [8] et [2], nous prouvons la convergence vers un unique équilibre stable.
\end{abstract}

\section{Version française abrégée.}

Nous étudions le comportement en temps grand de modèles de dynamique de populations. On considère un nombre fini de sous-populations, correspondant chacune à un trait ou type différent. Ces populations interagissent entre elles de façon compétitive. En notant $n_{i}(t)$ l'effectif de la sous-population numéro $i$, un des modèles les plus classiques est le système de Lotka-Volterra compétitif

$$
\frac{d}{d t} n_{i}=\left(r_{i}-\sum_{j} b_{i j} n_{j}\right) n_{i}, \quad i=1 \ldots N
$$

où $b_{i j} \geq 0$. On se place ici dans le cadre plus général du système

$\frac{d}{d t} n_{i}(t)=\left[r_{i}-\int_{\Omega} K_{i}(\alpha) L\left(\alpha, \sum_{j} B_{j}(\alpha) n_{j}(t)\right) d P(\alpha)\right] n_{i}(t), \quad i=1 \ldots N$

1. TOSCA project-team, INRIA Sophia Antipolis - Méditerranée, 2004 rte des Lucioles, BP. 93, 06902 Sophia Antipolis Cedex, France, E-mail : Nicolas.Champagnat@inria.fr

2. Laboratoire J.-A. Dieudonné, Université de Nice - Sophia Antipolis, Parc Valrose, 06108 Nice Cedex 02, France, E-mail : jabin@unice.fr

3. DAMTP, CMS, University of Cambridge, Wilberforce Road, Cambridge CB3 0WA, United Kingdom, Email : g.raoul@damtp.cam.ac.uk 
avec $(\Omega, P)$ un espace mesurable. Ce système peut s'interprêter comme un modèle avec ressources généralisées.

En utilisant les techniques développées dans [8] pour une version continue du premier modèle, et dans [2], on peut facilement montrer

Théorème Supposons que $\forall \alpha \in \Omega, L(\alpha, \cdot)$ est une fonction $C^{1}$ sur $\mathbb{R}$, positive sur $\mathbb{R}_{+}$, de dérivée $L^{\prime}(\alpha, \cdot)$ uniformément bornée en $\alpha$ sur tout compact de $\mathbb{R}$, que $L(\cdot, 0)$ est bornée, que $K$ et $B$ sont des fonctions positives bornées appartenant à $L^{1}(d P(\alpha))$ et que

(i) (Compétition stricte) Pour tout $\alpha \in \Omega, L(\alpha, \cdot)$ est strictement croissante et pour tout $1 \leq i \leq n, r_{i}<\int_{\Omega} K_{i}(\alpha) L(\alpha, \infty) d P(\alpha)$ où $L(\alpha, \infty):=$ $\lim _{x \rightarrow+\infty} L(\alpha, x) \in(0,+\infty]$.

(ii) (Symétrie) Il existe $C_{i}>0$ tq $B_{i}(\alpha)=C_{i} K_{i}(\alpha)$

(iii) (Non extinction) Pour tout $i, r_{i}>\int_{\Omega} K_{i}(d \alpha) L(\alpha, 0) d P(\alpha)$

(iv) (Non dégénérescence) Pour $I \subset\{1 \ldots N\}$, soit $\mathbb{R}^{I}$ l'ensemble des $n \in \mathbb{R}^{N}$

tels que $n_{i}=0$ pour tout $i \notin I$. Pour tout $I \subset\{1 \ldots N\}$ il y a au plus un $n \in \mathbb{R}^{I} t q$

$$
r_{i}-\int_{\Omega} K_{i}(\alpha) L\left(\alpha, \sum_{j=1}^{N} B_{j}(\alpha) n_{j}\right) d P(\alpha)=0, \quad \forall i \in I .
$$

Alors $\exists ! \bar{n}=\left(\bar{n}_{1}, \ldots, \bar{n}_{N}\right) \in \mathbb{R}_{+}^{N} \backslash\{0\}$, tel que pour toute solution $n(t)=$ $\left(n_{1}, \ldots, n_{N}\right)$ du modèle généralisé avec une donnée initiale $n_{i}(0)>0 \forall i$, on $a$

$$
n(t) \longrightarrow \bar{n}, \text { quand } t \rightarrow+\infty
$$

En particulier ce résultat implique

Proposition Supposons que $r_{i}>0$ pour tout $i$ et que la matrice $b_{i j}$ vérifie

$$
\exists C \in\left(\mathbb{R}_{+}^{*}\right)^{N} \text { tq } C_{i} b_{i j}=b_{j i} C_{j} \text {, et } \sum_{i j} u_{i} u_{j} b_{i j} C_{i}>0 \quad \forall u \in \mathbb{R}^{N} \backslash\{0\},
$$

Alors $\exists ! \bar{n}=\left(\bar{n}_{1}, \ldots, \bar{n}_{N}\right) \in \mathbb{R}_{+}^{N} \backslash\{0\}$ tel que pour toute solution $n(t)=$ $\left(n_{1}, \ldots, n_{N}\right)$ du premier modèle avec donnée initiale $n_{i}(0)>0 \forall i$,

$$
n(t) \longrightarrow \bar{n} \text {, quand } t \rightarrow+\infty \text {. }
$$

Nous obtenons également un résultat de convergence pour un systène classique en chémotaxie couplant dynamiques de populations et de resources. 


\section{Introduction}

We study the long time behaviour of models of population dynamics. We consider a finite number of subpopulations whose dynamics is governed by a system of competitive ODEs (in the sense of Hirsch, see e.g. [6]). We denote by $n_{i}(t), i=1 \ldots N$, the number of individuals of the subpopulation $i$.

The most classical models are competitive Lotka-Volterra equations

$$
\frac{d}{d t} n_{i}=\left(r_{i}-\sum_{j} b_{i j} n_{j}\right) n_{i}, \quad i=1 \ldots N,
$$

where $b_{i j} \geq 0$, and the models with a finite number of resources

$$
\frac{d}{d t} n_{i}=\left(-d_{i}+\sum_{k=1}^{K} I_{k} \eta_{k i}\right) n_{i}
$$

where $\eta_{k i} \geq 0$ and the $I_{k}$ are given by the Holling II functional response

$$
I_{k}=\frac{I_{k}^{0}}{1+\sum_{i=1}^{N} \mu_{k i} n_{i}} .
$$

This type of system appears in biology when one studies the dynamics of a system of interacting species (see $[7,5,9]$ ). It also appears in Trait Substitution Sequence models, where one considers a population structured by a continuous phenotype (see equation (1.5), (1.7) on this matter), where only a small number of traits are present (see $[10,1])$. These models have been used to develop the theory of Adaptative Dynamics (see [10, 1, 3]).

Previous asymptotic studies on this type of equations concern either very general properties (the existence of a carrying simplex [6]), or precise results but only for low dimensional systems $(N \leq 3[13])$, under strong assumptions of the coefficients (for instance, the matrix $\left(b_{i j}\right)$ is supposed to be diagonal dominant, see [7]), or only on local properties (the equilibrium population is locally stable, or populations $n_{i}$ do not vanish).

Note that both equations (1.1) and (1.2) may be interpreted as discrete versions of continuous models. To each subpopulation corresponds a phenotypic trait $x_{i} \in \mathbb{R}^{d}$, and then posing

$$
n(t, x)=\sum_{i=1}^{N} n_{i}(t) \delta_{x_{i}}
$$


one finds that Eq. (1.1) for instance is equivalent to

$$
\partial_{t} n(t, x)=\left(r(x)-\int_{\mathbb{R}^{d}} b(x, y) n(t, d y)\right) n(t, x),
$$

with $r_{i}=r\left(x_{i}\right)$ and $b_{i j}=b\left(x_{i}, x_{j}\right)$.

The long time behaviour of the continuous model (1.5) (with bounded initial data instead of Dirac masses) was studied in [8]. For a symmetric $b$ defining a positive operator, the convergence to the unique stable equilibrium was proved. For the case with resources, the result is essentially contained in [2], which generalizes the derivation of [4]. The derivation of such evolution models has recently been the subject of intense studies; while those are more complex than the relatively simple result presented here, an important step is often the study of the asymptotic in time of systems like (1.5). We refer for example to [11] and especially Chapter 2.

The study of the discrete or continuous models corresponds to slighty different biological questions; in the continuous case, it is for instance connected to the issue of speciation, or how from a continuum of traits a few well separated ones (the "species") are selected; in the discrete case, one is rather concerned about survival or extinction of each subpopulations. From a rigorous mathematical point of view, a result in the continuous case does not imply anything for the discrete one. However it is easy to apply the techniques developed in [8] and [2] to the discrete models; that is our aim.

First of all, we consider the very general equation

$$
\frac{d}{d t} n_{i}(t)=\left[r_{i}-\int_{\Omega} K_{i}(\alpha) L\left(\alpha, \sum_{j} B_{j}(\alpha) n_{j}(t)\right) d P(\alpha)\right] n_{i}(t), \quad i=1 \ldots N
$$

with $(\Omega, P)$ any measurable space, or in the continuous case

$$
\partial_{t} n(t, x)=\left[r(x)-\int_{\Omega} K(x, \alpha) L\left(\alpha, \int_{\mathbb{R}^{d}} B(y, \alpha) n(t, d y)\right) d P(\alpha)\right] n(t, x) .
$$

We prove the following

Theorem 1 Assume that $\forall \alpha \in \Omega, L(\alpha, \cdot)$ is $C^{1}$ on $\mathbb{R}$ and non negative on $\mathbb{R}_{+}$, with derivative $L^{\prime}(\alpha, \cdot)$ bounded on compact subsets of $\mathbb{R}$, uniformly in $\alpha \in \Omega$, that $L(\alpha, 0)$ is bounded, that $K$ and $B$ are bounded, non negative, in $L^{1}(d P(\alpha))$ and that 
(i) (Strict competition) For all $\alpha \in \Omega, L(\alpha, \cdot)$ is strictly increasing and $r_{i}<$ $\int_{\Omega} K_{i}(\alpha) L(\alpha, \infty) d P(\alpha)$ for all $1 \leq i \leq N$, where $L(\alpha, \infty):=\lim _{x \rightarrow+\infty} L(\alpha, x) \in$ $(0,+\infty]$.

(ii) (Symmetry) There exists $C_{i}>0$ s.t. $B_{i}(\alpha)=C_{i} K_{i}(\alpha)$

(iii) (Non extinction) For any $i, r_{i}>\int_{\Omega} K_{i}(\alpha) L(\alpha, 0) d P(\alpha)$

(iv) (Non degeneracy) For any $I \subset\{1 \ldots N\}$, let $\mathbb{R}^{I}$ be the set of $n \in \mathbb{R}^{N}$ s.t. $n_{i}=0$ for all $i \notin I$. For all $I \subset\{1 \ldots N\}$, there exists at most one $n \in \mathbb{R}^{I}$ s.t.

$$
r_{i}-\int_{\Omega} K_{i}(\alpha) L\left(\alpha, \sum_{j=1}^{N} B_{j}(\alpha) n_{j}\right) d P(\alpha)=0, \quad \forall i \in I .
$$

Then there exists a unique $\bar{n}=\left(\bar{n}_{1}, \ldots, \bar{n}_{N}\right) \in \mathbb{R}_{+}^{N}$ with $\bar{n} \neq 0$, s.t. for any solution $n(t)=\left(n_{1}, \ldots, n_{N}\right)$ to (1.6) with initial data $n_{i}(0)>0$ for any $i$,

$$
n(t) \longrightarrow \bar{n} \text {, as } t \rightarrow+\infty \text {. }
$$

Note that, if one had $r_{i}>\int K_{i}(\alpha) L(\alpha, \infty) d P(\alpha)$ for some $i$, then $n_{i}(t) \rightarrow$ $+\infty$ if $n_{i}(0)>0$. Assumption $(i)$ hence ensures the non-explosion of the system.

Eq. (1.6) could be directly derived from simple biological considerations. It assumes that the reproduction rate of a population of type $i$ (or with trait $x_{i}$ ) is the difference between a fixed rate depending only on the trait and a competitive interaction with the other populations, resulting from the interaction with the environment. The state of each component of this environment (indicated by different values of $\alpha$ ) is given by the sum

$$
\sum_{j} B_{j}(\alpha) n_{j}(t)
$$

Each such component has some independent effect on the reproduction. To get the total reproduction rate one sums over those.

Eq. (1.6) is hence an obvious generalization, with a possibly infinite number of resources, of the model (1.2). It also contains the Lotka-Volterra system (1.1). In this case, Theorem 1 gives

Proposition 1 Assume that $r_{i}>0$ for all $i$ and that the matrix $b_{i j}$ satisfies

$$
\exists C \in\left(\mathbb{R}_{+}^{*}\right)^{N} \text { s.t. } C_{i} b_{i j}=b_{j i} C_{j} \text {, and } \sum_{i j} u_{i} u_{j} b_{i j} C_{i}>0 \quad \forall u \in \mathbb{R}^{N} \backslash\{0\} \text {, }
$$


then there exists a unique $\bar{n}=\left(\bar{n}_{1}, \ldots, \bar{n}_{N}\right) \in \mathbb{R}_{+}^{N}$ with $\bar{n} \neq 0$, s.t. for any solution $n(t)=\left(n_{1}, \ldots, n_{N}\right)$ to (1.1) with initial data $n_{i}(0)>0$ for any $i$,

$$
n(t) \longrightarrow \bar{n} \text {, as } t \rightarrow+\infty \text {. }
$$

This result shows that, in Lotka-Volterra systems which are symmetric in the sense of (1.9), the competition between a mutant trait and a resident population leads to a unique stationary state, regardless of the initial population state. This is precisely the assumption needed in [1] to apply a limit of large population and rare mutations to an individual-based model. In particular, Thm. 2.7 of [1] applies to symmetric competitive Lotka-Volterra systems.

Finally the same techniques and Lyapunov functionals also apply to some systems where the ressources themselves solves a differential equation. This is in particular the case of the so-called "chemostat" which consists of the system composed of

$$
\frac{d}{d t} n_{i}=\left(-d_{i}+\sum_{k=1}^{K} f_{k}\left(I_{k}\right) \eta_{k i}\right) n_{i}
$$

coupled with

$$
\frac{d}{d t} I_{k}=I_{k}^{0}-I_{k}-f_{k}\left(I_{k}\right) \sum_{i=1}^{N} \mu_{k i} n_{i}, \quad I_{k}(0) \geq 0 .
$$

Note that if $f_{k}=I d$ and if one assumes that the ressources adapt themselves faster than the individuals and hence take $d I_{k} / d t=0$ in (1.11), one recovers (1.2)-(1.3).

The coupled system (1.10)-(1.11) was introduced and studied in [12] together with other of the same types but only with one ressource: $K=1$. However adapting the proof of Th. 1, one can show for any number of ressources

Theorem 2 Assume that the $I_{k}^{0}$ are positive, and that

(i) (Strict competition) $f_{k}$ is $C^{1}$ with $f_{k}^{\prime}>0$ on $\mathbb{R}_{+}, f_{k}(0)=0$ and $\eta_{k i}>0$ for all $1 \leq i \leq N, 1 \leq k \leq K$

(ii) (Symmetry) There exists $C_{i}>0$ s.t. $\mu_{k i}=C_{i} \eta_{k i}$

(iii) (Non explosion, non extinction) $\forall i, \sum_{k} \eta_{k i} f_{k}(0)<d_{i}<\sum_{k=1}^{K} \eta_{k i} f_{k}\left(I_{k}^{0}\right)$

(iv) (Non degeneracy) For any $I \subset\{1 \ldots N\}$, let $\mathbb{R}^{I}$ be the set of $n \in \mathbb{R}^{N}$ s.t. 
$n_{i}=0$ for all $i \notin I$. For all $I \subset\{1 \ldots N\}$, there exists at most one $n \in \mathbb{R}^{I}$ s.t.

$$
d_{i}-\sum_{k=1}^{K} f_{k}\left(\psi_{k}\left(\sum_{i=1}^{N} \mu_{k i} n_{i}\right)\right) \eta_{k i}=0, \quad \forall i \in I,
$$

where $\psi_{k}(n)$ is the inverse function of $\left(I_{k}^{0}-x\right) / f_{k}(x)$ on $\left[0, I_{k}^{0}\right]$.

Then there exists a unique $(\bar{n}, \bar{I})=\left(\bar{n}_{1}, \ldots, \bar{n}_{N}, \bar{I}_{1}, \ldots, \bar{I}_{K}\right) \in \mathbb{R}_{+}^{N+K}$ with $\bar{n} \neq 0$, s.t. for any solution $(n(t), I(t))$ to (1.10) and (1.11) with initial data $n_{i}(0)>0$ for any $i$,

$$
(n(t), I(t)) \longrightarrow(\bar{n}, \bar{I}), \text { as } t \rightarrow+\infty .
$$

\section{Proof of Prop. 1}

Define the matrix $m_{i j}=C_{i} b_{i j}$. Note that $m$ is symmetric and positive definite. Hence there exists an orthonormal basis of eigenvectors $U^{i}, i=$ $1 \ldots N$, and corresponding eigenvalues $\lambda_{i}>0$.

Then put $L(\alpha, \cdot)=\mathrm{Id}, \Omega=\{1, \ldots, N\}, P=\frac{1}{N} \sum_{i=1}^{N} \delta_{i}, B_{j}(\alpha)=\sqrt{\lambda_{\alpha}} U_{j}^{\alpha}$, $K_{i}(\alpha)=C_{i}^{-1} \sqrt{\lambda_{\alpha}} U_{i}^{\alpha}$ and note that

$$
\begin{aligned}
\sum_{j=1}^{N} b_{i j} n_{j} & =\frac{1}{C_{i}} \sum_{j=1}^{N} m_{i j} n_{j}=\frac{1}{C_{i}}[M n]_{i} \\
& =\frac{1}{C_{i}}\left[M\left(\sum_{\alpha} U^{\alpha}\left\langle U^{\alpha}, n\right\rangle\right)\right]_{i}=\frac{1}{C_{i}} \sum_{\alpha=1}^{N} \lambda_{\alpha} U_{i}^{\alpha}\left(\sum_{j=1}^{N} U_{j}^{\alpha} n_{j}\right) \\
& =\int_{\Omega} K_{i}(\alpha) L\left(\sum_{j=1}^{N} B_{j}(\alpha) n_{j}\right) d P(\alpha) .
\end{aligned}
$$

Therefore Eq. (1.6) indeed yields (1.1) in that particular case.

Conditions $(i)$ and $(i i)$ of Theorem 1 are obviously satisfied. Conditions (iii) holds since $r_{i}>0$ for all $i$ and $L(\alpha, 0)=0$. As for condition (iv), assume that for a subset $I$ one has two vectors $n_{j}^{\gamma}, \gamma=1,2$, s.t. $n_{i}^{\gamma}=0$ for $i \notin I$ and

$$
r_{i}=\int_{\Omega} K_{i}(\alpha) L\left(\alpha, \sum_{j=1}^{N} B_{j}(\alpha) n_{j}^{\gamma}\right) d P(\alpha)=\sum_{j=1}^{N} b_{i j} n_{j}^{\gamma} \quad \forall i \in I .
$$

Put $\delta n=n^{1}-n^{2}$ and simply note that $\delta n_{i} \sum_{j=1}^{N} b_{i j} \delta n_{j}=0$ pour $i=1 \ldots N$. 
This means that $\delta n=0$ and proves $(i v)$ since $\sum_{i, j=1}^{N} C_{i} b_{i j} \delta n_{i} \delta n_{j}=0$. Hence the proposition is implied by Theorem 1 .

Note that the same argument works in the continuous case and Eq. (1.5) is a particular case of (1.7) for $x \in O$ a bounded domain. The condition on $b$ is

$$
C(x) b(x, y)=C(y) b(y, x), \quad \int_{O^{2}} C(x) b(x, y) n(x) n(y) d x d y>0 \quad \forall n \neq 0 .
$$

One still puts $L(\xi)=\xi$. Notice that $C(x) b(x, y)$ defines a compact, selfadjoint and positive operator on $L^{2}(O)$. Diagonalizing the operator, one gets

$$
C(x) b(x, y)=\sum_{\alpha} \lambda_{i} f_{\alpha}(x) f_{\alpha}(y)
$$

with $\lambda_{\alpha}>0$ the eigenvalues, tending to $+\infty$ and $f_{\alpha}$ the corresponding normalized eigenvector. It is hence enough to take $\Omega=\mathbb{N}$ and $K(x, \alpha)=$ $\sqrt{\lambda_{\alpha}} f_{\alpha}(x)$.

In the particular case where $b(x, y)=b(x-y)$ on the whole $\mathbb{R}^{d}$ and $C=1$, by Fourier transform, the condition on $b$ means that $\hat{b}>0$. One then takes $\Omega=\mathbb{R}^{d}$ and

$$
K(x, \alpha)=(\cos (\alpha \cdot x) \sqrt{\hat{b}(\alpha)}, \sin (\alpha \cdot x) \sqrt{\hat{b}(\alpha)}) .
$$

\section{Proof of Theorem 1}

The proof is based on the study of the following Lyapunov functional

$$
F(n)=\int_{\Omega} H\left(\alpha, \sum_{j=1}^{N} B_{j}(\alpha) n_{j}\right) d P(\alpha)-\sum_{i=1}^{N} C_{i} r_{i} n_{i}
$$

where for all $\alpha \in \Omega, H(\alpha, \cdot)$ is the antiderivative of $L(\alpha, \cdot)$ such that $H(\alpha, 0)=$ 0 and hence strictly convex.

\section{1 $F$ is a strict Lyapunov functional}

Let $n$ be a solution to (1.6). Then by a direct computation

$$
\frac{d}{d t} F(n(t))=-\sum_{i=1}^{N} C_{i} n_{i}\left[\int_{\Omega} K_{i}(\alpha) L\left(\alpha, \sum_{j=1}^{N} B_{j}(\alpha) n_{j}\right) d P(\alpha)-r_{i}\right]^{2} .
$$


Therefore $F(n(t))$ is non increasing and its derivative in time vanishes only on stationary solutions to (1.6), i.e. $F$ is a strict Lyapunov functional for the system (1.6).

Thanks to condition $(i)$,

$$
\frac{\partial F}{\partial n_{i}} \geq C_{i}\left(\int_{\Omega} K_{i}(\alpha) L\left(\alpha, \frac{K_{i}(\alpha)}{C_{i}} n_{i}\right) d P(\alpha)-r_{i}\right) \geq a>0
$$

if $n_{i}$ is large enough. Therefore, there is a constant $a^{\prime}>0$ s.t. $\nabla F(n)$. $n \geq a^{\prime}\|n\|$ if $\|n\|$ is large enough. This implies that $F(n) \rightarrow+\infty$ when $\|n\| \rightarrow+\infty$, and entails that $n(t)$ is uniformly bounded.

Let $n \in \mathbb{R}_{+}^{N}$ be a steady-state of (1.6) and let $I$ be the set of $i$ s.t. $n_{i}>0$. Then, for any $i \in I$ one needs to have

$$
\int_{\Omega} K_{i}(\alpha) L\left(\alpha, \sum_{j=1}^{N} B_{j}(\alpha) n_{j}\right) d P(\alpha)=r_{i} .
$$

By condition $(i v)$ there is at most one such solution for every $I$, and there are only a finite number of possible $I, F$ has then a finite number of steady-states.

Classical Lyapunov functionnals' techniques then entail that the solution $n(t)$ to (1.6) converges to a steady-state $\tilde{n}$ for any initial condition $n(0)$.

\subsection{The functional $F$ is convex}

Compute

$$
\frac{\partial^{2} F}{\partial n_{i} \partial n_{k}}=\int_{\Omega} B_{i}(\alpha) B_{k}(\alpha) L^{\prime}\left(\alpha, \sum_{j=1}^{N} B_{j}(\alpha) n_{j}\right) d P(\alpha) .
$$

Hence as $L$ is increasing

$$
\sum_{i, k} \frac{\partial^{2} F}{\partial n_{i} \partial n_{k}} \xi_{i} \xi_{k}=\int_{\Omega}\left(\sum_{i} \xi_{i} B_{i}(\alpha)\right)^{2} L^{\prime}\left(\alpha, \sum_{j=1}^{N} B_{j}(\alpha) n_{j}\right) d P(\alpha) \geq 0 .
$$

$F$ is therefore convex and any local minimum on $\mathbb{R}_{+}^{N}$ is global.

Since (1.6) has a finite number of stationary solutions, this clearly implies that $F$ admits a unique global minimizer $\bar{n}$. Otherwise, $F$ would reach its minimum on the whole segment linking two distinct minimizers.

The object of the next subsection is to prove that $\bar{n}$ satisfies a stronger property: this is the unique ESS of the system. 


\subsection{Uniqueness of the ESS}

Any local minimizer $n \in \mathbb{R}_{+}^{N}$ of the functional $F$ necessarily satisfies

$$
\begin{aligned}
& \int_{\Omega} K_{i}(\alpha) L\left(\alpha, \sum_{j=1}^{N} B_{j}(\alpha) n_{j}\right) d P(\alpha)=r_{i}, \quad \forall i \text { s.t. } n_{i}>0, \\
& \int_{\Omega} K_{i}(\alpha) L\left(\alpha, \sum_{j=1}^{N} B_{j}(\alpha) n_{j}\right) d P(\alpha) \geq r_{i}, \quad \forall i \text { s.t. } n_{i}=0 .
\end{aligned}
$$

This condition corresponds to the usual definition of an Evolutionarily Stable Strategy in adaptive dynamics (see for instance [3]). It turns out that there exists at most one ESS, $\bar{n}$. Hence being an ESS is a necessary and sufficient condition to be the global minimizer of $F$.

Indeed take two $n^{\gamma} \in \mathbb{R}_{+}^{N}, \gamma=1,2$ satisfying (3.1) and compute

$$
\begin{aligned}
0 \geq \sum_{i} C_{i} n_{i}^{1}\left(r_{i}-\int_{\Omega} K_{i}(\alpha) L\left(\alpha, \sum_{j=1}^{N} B_{j}(\alpha) n_{j}^{2}\right) d P(\alpha)\right) \\
\quad+\sum_{i} C_{i} n_{i}^{2}\left(r_{i}-\int_{\Omega} K_{i}(\alpha) L\left(\alpha, \sum_{j=1}^{N} B_{j}(\alpha) n_{j}^{1}\right) d P(\alpha)\right) .
\end{aligned}
$$

This last quantity is equal to (thanks to (3.1))

$$
\begin{aligned}
& \sum_{i} C_{i}\left(n_{i}^{1}-n_{i}^{2}\right)\left(r_{i}-\int_{\Omega} K_{i}(\alpha) L\left(\alpha, \sum_{j=1}^{N} B_{j}(\alpha) n_{j}^{2}\right) d P(\alpha)\right) \\
& \quad+\sum_{i} C_{i}\left(n_{i}^{2}-n_{i}^{1}\right)\left(r_{i}-\int_{\Omega} K_{i}(\alpha) L\left(\alpha, \sum_{j=1}^{N} B_{j}(\alpha) n_{j}^{1}\right) d P(\alpha)\right)
\end{aligned}
$$

and to

$$
\begin{aligned}
\int_{\Omega} & \left(\sum_{j} B_{j}(\alpha) n_{j}^{1}-\sum_{j} B_{j}(\alpha) n_{j}^{2}\right) \\
& \left(L\left(\alpha, \sum_{j=1}^{N} B_{j}(\alpha) n_{j}^{1}\right)-L\left(\alpha, \sum_{j=1}^{N} B_{j}(\alpha) n_{j}^{1}\right)\right) d P(\alpha) .
\end{aligned}
$$

As $L(\alpha, \cdot)$ is strictly increasing, this implies that for $P$ a.e. $\alpha, \sum_{i=1}^{N} B_{i}(\alpha)\left(n_{i}^{1}-\right.$ $\left.n_{i}^{2}\right)=0$ and by $(i v)$, it means that $n^{1}=n^{2}$. 


\subsection{Conclusion of the proof of Thm. 1}

Assume that $n_{i}(0)>0$ for all $1 \leq i \leq N$. We know from Subsection 3.1 that $n(t)$ converges to a steady-state $\tilde{n}$ when $t \rightarrow \infty$.

If $\tilde{n}$ does not satisfy (3.1), there exists $i \in\{1, \ldots, N\}$ such that

$$
\lambda_{i}:=r_{i}-\int_{\Omega} K_{i}(\alpha) L\left(\alpha, \sum_{j=1}^{N} B_{j}(\alpha) \tilde{n}_{j}\right) d P(\alpha)>0 .
$$

Since $n_{i}(0)>0, n_{i}>0$ at all times, and the linearized equation around $\tilde{n}$ shows that $n$ cannot converge to $\tilde{n}$ :

$$
\begin{aligned}
\frac{d}{d t}(n-\tilde{n})_{i} & =\left(\lambda_{i}+O(\|n-\tilde{n}\|)\right)(n-\tilde{n})_{i} \\
& \geq \frac{\lambda_{i}}{2}(n-\tilde{n})_{i},
\end{aligned}
$$

provided that $\|n-\tilde{n}\|$ is small enough.

Therefore, $\tilde{n}=\bar{n}$, and the proof of Thm. 1 is completed.

\section{Proof of Theorem 2}

First note that the equilibria $(\bar{n}, \bar{I})$ of $(1.10)-(1.11)$ are exactly those of

$$
\frac{d}{d t} n_{i}=\left[-d_{i}+\sum_{k=1}^{K} \eta_{k i} f_{k}\left(\psi_{k}\left(\sum_{i=1}^{N} \mu_{k i} n_{i}\right)\right)\right] n_{i},
$$

coupeld with $\bar{I}_{k}=\psi_{k}\left(\sum_{i} \mu_{k i} \bar{n}_{i}\right)$. Taking $\Omega=\{1, \ldots, K\}, L(\alpha, x)=f_{\alpha}\left(I_{\alpha}^{0}\right)-$ $f_{\alpha}\left(\psi_{\alpha}(x)\right)$ and $P(d \alpha)=\sum_{k=1}^{K} \delta_{k}(d \alpha)$, (4.1) has exactly the form of (1.6). Therefore the definition of the ESS, its existence and uniqueness are exactly the same as in the proof of Theorem 1.

Let us denote $(\bar{n}, \bar{I})$ the unique ESS of the system. The exact equivalent of the functional $F$ would be

$$
\tilde{F}(n, I)=\sum_{i=1}^{N} C_{i}\left(n_{i}-\bar{n}_{i} \log n_{i}\right)+\sum_{k=1}^{K}\left(I_{k}-f_{k}\left(\bar{I}_{k}\right) g_{k}\left(I_{k}\right)\right),
$$

with $g_{k}$ an antiderivative of $1 / f_{k}$ on $(0,+\infty)$. Note that $\tilde{F}$ is well-defined if $n_{i}>0$ for $i$ such that $\bar{n}_{i}>0$. Let us consider a solution of (1.10)- (1.11) 
with such an initial condition. Then $n_{i}(t)>0$ for all $t \geq 0$ and $i$ such that $\bar{n}_{i}>0$, and we compute

$$
\begin{aligned}
\frac{d}{d t} \tilde{F}(n(t), I(t)) & =\sum_{i}\left(n_{i}-\bar{n}_{i}\right)\left(\sum_{k} C_{i} \eta_{k i} f_{k}\left(I_{k}\right)-C_{i} d_{i}\right) \\
& +\sum_{k} \frac{f_{k}\left(I_{k}\right)-f_{k}\left(\bar{I}_{k}\right)}{f_{k}\left(I_{k}\right)}\left(I_{k}^{0}-I_{k}-f_{k}\left(I_{k}\right) \sum_{i} \mu_{k i} n_{i}\right)
\end{aligned}
$$

Recalling that $(\bar{n}, \bar{I})$ is a stationary solution, one gets

$$
\begin{aligned}
\frac{d \tilde{F}}{d t} & =\sum_{i} \sum_{k}\left(n_{i}-\bar{n}_{i}\right) \mu_{k i}\left(f_{k}\left(I_{k}\right)-f_{k}\left(\bar{I}_{k}\right)\right)+\sum_{i}\left(n_{i}-\bar{n}_{i}\right)\left(\sum_{k} \mu_{k i} f_{k}\left(\bar{I}_{k}\right)-C_{i} d_{i}\right) \\
& -\sum_{k} \sum_{i} \mu_{k i}\left(n_{i}-\bar{n}_{i}\right)\left(f_{k}\left(I_{k}\right)-f_{k}\left(\bar{I}_{k}\right)\right) \\
& +\sum_{k} \frac{f_{k}\left(I_{k}\right)-f_{k}\left(\bar{I}_{k}\right)}{f_{k}\left(I_{k}\right)}\left(I_{k}^{0}-I_{k}-f_{k}\left(I_{k}\right) \sum_{i} \mu_{k i} \bar{n}_{i}\right)
\end{aligned}
$$

As $(\bar{n}, \bar{I})$ is an ESS then

$$
\left(n_{i}-\bar{n}_{i}\right)\left(\sum_{k} \mu_{k i} f_{k}\left(\bar{I}_{k}\right)-C_{i} d_{i}\right)=n_{i}\left(\sum_{k} \mu_{k i} f_{k}\left(\bar{I}_{k}\right)-C_{i} d_{i}\right) \leq 0 .
$$

Finally, recalling that $\sum_{i} \mu_{k i} \bar{n}_{i}=\left(I_{k}^{0}-\bar{I}_{k}\right) / f_{k}\left(\bar{I}_{k}\right)$

$$
\frac{d \tilde{F}}{d t} \leq-\sum_{k} \frac{\left(f_{k}\left(I_{k}\right)-f_{k}\left(\bar{I}_{k}\right)\right)^{2}}{f_{k}\left(I_{k}\right)} \sum_{i} \mu_{i k} \bar{n}_{i}-\sum_{k} \frac{f_{k}\left(I_{k}\right)-f_{k}\left(\bar{I}_{k}\right)}{f_{k}\left(I_{k}\right)}\left(I_{k}-\bar{I}_{k}\right),
$$

which is indeed a Lyapunov functional as the $f_{k}$ are increasing. Note that the right-hand side vanishes iff $I_{k}=\bar{I}_{k}$ for all $k$. In that sense this Lyapunov functional is degenerate as now the $I_{k}$ are not anymore connected to the $n_{i}$ by an explicit relation.

As we are in a finite dimension setting, it is easy to correct this problem. Consider instead of $\tilde{F}$

$$
G=\tilde{F}+\gamma \sum_{k}\left(I_{k}-\bar{I}_{k}\right) \sum_{i} \mu_{k i}\left(n_{i}-\bar{n}_{i}\right) .
$$

Note that, since

$$
\frac{d}{d t}\left(\sum_{i} C_{i} n_{i}+\sum_{k} I_{k}\right) \leq a-b\left(\sum_{i} C_{i} n_{i}+\sum_{k} I_{k}\right)
$$


for explicit constants $a, b>0$, all solutions to (1.10)- (1.11) enter in finite time the set $D=\left\{\sum_{i} C_{i} n_{i}+\sum_{k} I_{k} \leq a / b, I_{k} \geq c, \forall k \leq K\right\}$, where $c>0$ is such that $c+f(c) b / a=\min _{k} I_{k}^{0}$.

Taking $\gamma$ small enough and using the fact that $f_{k}^{\prime}$ is uniformly bounded from below, one simply gets that, on the set $D$,

$$
\begin{aligned}
\frac{d G}{d t} & \leq-\frac{1}{2} \sum_{k} \frac{\left(f_{k}\left(I_{k}\right)-f_{k}\left(\bar{I}_{k}\right)\right)^{2}}{f_{k}\left(I_{k}\right)} \sum_{i} \mu_{k i} \bar{n}_{i}-\sum_{k} \frac{f_{k}\left(I_{k}\right)-f_{k}\left(\bar{I}_{k}\right)}{f_{k}\left(I_{k}\right)}\left(I_{k}-\bar{I}_{k}\right) \\
& -\frac{\gamma}{2} \sum_{k} f_{k}\left(I_{k}\right)\left(\sum_{i} \mu_{k i}\left(n_{i}-\bar{n}_{i}\right)\right)^{2} .
\end{aligned}
$$

By assumption $(i v)$, this vanishes only if $(n, I)=(\bar{n}, \bar{I})$. Theorem 2 now simply follows from Lyapunov's Theorem.

Acknowledgments: The first author is grateful to Michel Benaïm for useful discussions on the dynamical systems context of the problem. GR has been supported by Award No. KUK-I1-007-43 of Peter A. Markowich, made by King Abdullah University of Science and Technology (KAUST).

\section{References}

[1] N. Champagnat, S. Méléard, Polymorphic evolution sequence and evolutionary branching. To appear in Probab. Theor. Relat. Fields (2010).

[2] N. Champagnat, P.E. Jabin, The evolutionary limit for models of populations interacting competitively with many resources. Preprint (2010).

[3] O. Diekmann, A beginner's guide to adaptive dynamics. Banach Center Publications 63, 47-86, (2004).

[4] O. Diekmann, P.E. Jabin, S. Mischler, B. Perthame, The dynamics of adaptation: An illuminating example and a Hamilton-Jacobi approach. Theor. Popul. Biol. 67, 257-271, (2005).

[5] K. Gopalsamy, Global asymptotic stability in Volterra's population systems. J. Math. Biology 19, 157-168, (1984).

[6] M.W. Hirsch, Systems of differential equations which are competitive or cooperative. III. Competing species. Nonlinearity 1(1), 51-71 (1988).

[7] Hofbauer and Sigmund, Evolutionary Games and Population Dynamics, Cambridge University Press, Cambridge, 1998. 
[8] P.E. Jabin, G. Raoul, Selection dynamics with competition, to appear in J. Math. Biol.

[9] K. Krisztina, S. Kovács Qualitative behavior of $n$-dimensional ratiodependent predator-prey systems. Appl. Math. Comput. 199(2), 535$546,(2008)$.

[10] J.A.J. Metz, S.A.H. Geritz, G. Meszéna, F.A.J. Jacobs, J.S. van Heerwaasden, Adaptive dynamics: a geometrical study of the consequences of nearly faithful reproduction. In S. J. van Strien and S. M. Verduyn Lunel, editors, Stochastic and Spatial Structures of Dynamical Systems, 183-231, Amsterdam, 1996.

[11] B. Perthame, Transport Equations in Biology. Frontiers in Mathematics Birkhäuser 2007.

[12] H.L. Smith and P. Waltman, The Theory of the Chemostat, Dynamics of Microbial Competition. Cambridge studies in Mathematical Biology 13, Cambridge University Press 1995.

[13] M.L. Zeeman, Hopf bifurcations in competitive three-dimensional Lotka Volterra systems. Dynam. Stability Systems 8(3), 189-217 (1993). 